\title{
$\beta$-HYDROXYBUTYRATE AND RESPONSE TO HYPOXIA IN THE GROUND SQUIRREL, SPERMOPHILUS TRIDECIMLINEATUS
}

\author{
Louis G. D’Alecy, ${ }^{*} \dagger$ Edward F. Lundy, $†$ Matthew J. Kluger, $\ddagger$ Christian T. Harker, $\ddagger$ \\ Daniel R. LeMay $\ddagger$ and Marshal Shlafer $\S$ \\ †Departments of Physiology, and Surgery; $\ddagger$ Department of Physiology; and §Departments of \\ Pharmacology, and Surgery, The University of Michigan School of Medicine, \\ Ann Arbor, MI, USA
}

(Received 12 October 1989)

\begin{abstract}
Previous studies have suggested that elevated ketone levels are associated with increased survival time in rodents exposed to hypoxia. In this study the association between whole blood BHB $(\beta$-hydroxybutyrate) and hypoxic survival time was investigated in hibernating and non-hibernating ground squirrels and in rats.

2. Non-hibernating ground squirrels and rats were exposed to hypoxia $\left(4.5 \% \mathrm{O}_{2}\right)$. One hundred per cent of ground squirrels survived $1 \mathrm{hr}$ of hypoxia vs $20 \%$ of rats.

3. Ketone levels were significantly higher in ground squirrels than rats during hypoxia, and rats surviving the longest had the highest ketone levels.

4. When hibernation was induced in ground squirrels there was a significant increase in $\beta$-hydroxybutyrate from 0.45 to $1.6 \mathrm{mM}(P=0.0005)$.

5. Ground squirrel heart mitochondrial respiratory control ratios and ATP synthesis rates indicated no preferential ketone utilization which might suggest a possible extramitochondrial role of BHB during hypoxia.

6. We conclude that elevated blood BHB levels are associated with increased hypoxic survival and they may have evolved in response to life-threatening hypoxia as experienced during hibernation.
\end{abstract}

\section{INTRODUCTION}

Hypoxia is not tolerated well by laboratory rodents, however several studies from our laboratory using mice and rats have demonstrated that drug-induced systemic ketosis is associated with a dramatic increase in hypoxic tolerance (Eiger et al., 1980, Kirsch et al., 1980, Lundy et al., 1984). In addition, several naturally occurring conditions, including fasting (Owen $e t$ al., 1967), neonatal life (Himwhich et al., 1942), and untreated diabetes (Gottstein et al., 1972), results in elevations in blood ketone levels. Experimentally fasted and alloxan diabetic mice have been shown to have an increase in hypoxic tolerance (Kirsch and D'Alecy, 1979). This led us to speculate that the ability to elevate blood BHB may play an adaptive role in life-threatening hypoxia. Hibernating ground squirrels (Spermophilus tridecemilineatus) in their fossorial habitat have been reported to experience hypoxia, particularly during arousal from hibernation (Bullard et al., 1960; Hiestand et al., 1950; Lyman et al., 1982). The aims of this study were to determine: (1) the ability of ground squirrels to tolerate hypoxia as compared to rats: (2) the effect of hypoxia on whole blood BHB levels; and (3) the effect of hibernation on blood BHB levels. Mitochondrial function was studied to determine if there was

*Author to whom all correspondence should be addressed at: Department of Physiology, The University of Michigan, 7799 Medical Science Building II, Ann Arbor, MI 48109, USA (Tel: 313763 2566). preferential BHB utilization by ketotic hibernating ground squirrels.

\section{METHODS}

\section{Experimental Animals}

Forty-six 100-200 g 13-lined ground squirrels (Spermophilus tridecimilineatus) were obtained during the summer months from the vicinity of Ann Arbor, MI, housed in groups of three to five, and fed a diet of sunflower seeds, fresh fruit, vegetables, and tap water ad libitum. Seventyfour, 250-390 g male Sprague-Dawley rats (Charles Rivers) were individually housed with tap water and rat chow ad libitum. Experiments conformed with the principles of the Guide for the Care and Use of Experimental Animals (US Department of Health and Human Services).

\section{Hypoxia experiments}

All hypoxic tolerance tests were done at $22 \pm 1{ }^{\circ} \mathrm{C}$. Animals were exposed to hypoxia in a clear plastic tube measuring $30 \mathrm{~cm}$ in length and $10 \mathrm{~cm}$ in diameter, with large rubber stoppers at each end. The chamber was connected via polyethlene tubing to $20.9 \%$ (room air) or $4.5 \% \mathrm{O}_{2}$ in nitrogen (pre-mixed gas). Continuous monitoring of the chamber oxygen tension and temperature was performed using an oxygen/temperature analyzer (YSI 2600). Nonhibernating ground squirrels and Sprague-Dawley rats were placed in the chamber and exposed to $4.5 \% \mathrm{O}_{2}$ for 5,10 , and $60 \mathrm{~min}$. At the end of the specified period the animals were removed from the chamber, decapitated, and blood obtained for BHB, glucose, glucagon, and insulin measurements as previously described (Lundy et al., 1987). Eight of ten rats that were exposed to hypoxia for $60 \mathrm{~min}$ died before the $60 \mathrm{~min}$ had elapsed. At the moment spontaneous ventilation stopped the rat was removed from the chamber and 
immediately decapitated to obtain blood. Hypoxic survival time refers to the time from the onset of hypoxia to the cessation of spontaneous ventillation. A previous study (Kirsch et al., 1980) indicated that the hearts of mice are still beating at the moment of ventilatory arrest and therefore does not indicate the exact time of brain death. However the moment of ventilatory arrest is a simple and consistently reproducible marker of hypoxic intolerance (Eiger et al., 1980; Kirsch and D'Alecy 1979; Kirsch et al., 1980; Lundy et al. 1984).

\section{Temperature and blood studies}

Non-hibernating ground squirrels $(n=18)$ and rats ( $n=16$ ) were housed at $10 \pm 1^{\circ} \mathrm{C}$ for $48 \mathrm{hr}$ to determine the effects of cold stress on whole blood BHB, glucose, glucagon and insulin. Control groups of ground squirrels $(n=3)$ and rats $(n=21)$ were housed at $22 \pm 1^{\circ} \mathrm{C}$ and tested for the same blood contents. To induce hibernation, ground squirrels were kept at $10 \pm 1^{\circ} \mathrm{C}$ until hibernation began, usually after 7-60 days. Blood for analysis was obtained by decapitation to collect adequate volume. Core body temperature was measured using a Yellow Springs Telethermometer with a needle themistor inserted into the thorax immediately after decapitation.

\section{Mitochondrial studies}

Respiratory control ratios (RCR) and oxidative phosphorylation rates (OPR) were determined from mitochondria isolated from the hearts of hibernating and non-hibernating ground squirrels (D'Alecy et al., 1986). The RCR was calculated as the quotient of the State 3 rate of oxygen consumption (ADP present) and the subsequent State 4 rate (ADP absent). The OPR was calculated as the product of State 3 respiratory rate and the accompanying ADP:O ratio. The OPR estimates the rate of mitochondrial ATP synthesis, normalized per mg of mitochondrial protein. Oxygen consumption was measured and computed according to the method of Chance and Williams (1956).

\section{Statistics}

Statistical analysis was performed using the Michigan Interactive Data Analysis System (MIDAS) on an Amdahl 470/V6 computer. Statistical comparisons between ground squirrel and rat blood BHB concentrations were performed using Student's $t$-test. Survival rate analyses were with
PERCENT SURVIVAL VS. HYPOXIC EXPOSURE TIME

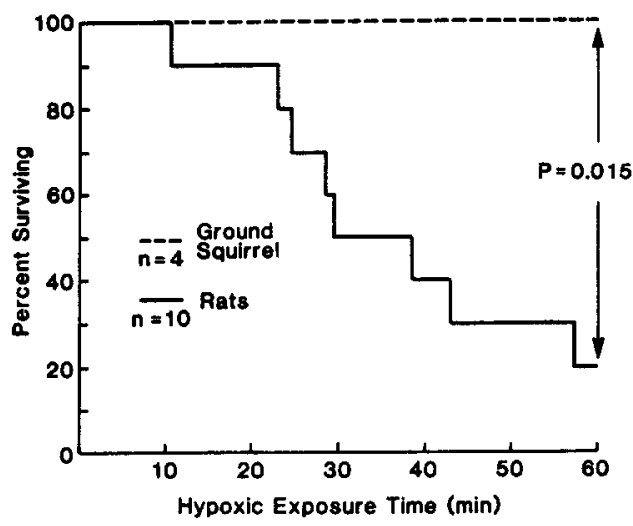

Fig. 1. Per cent survival over $1 \mathrm{hr}$ of hypoxia $\left(4.5 \% \mathrm{O}_{2}\right)$ in rats and non-hibernating ground squirrels. Rats have a significantly lower hypoxic tolerance compared to $100 \%$ survival for ground squirrels (Fisher's Exact test). Arrest of spontaneous ventilation is used as a reproducible indicator of hypoxic intolerance.

Fisher's Exact test. All values are expressed as mean \pm SEM with sample size designated $n$.

\section{RESULTS}

The first set of experiments involved non-hibernating ground squirrels. One hundred per cent of the ground squirrels survived $60 \mathrm{~min}$ of exposure to hypoxia (Fig. 1). Rats had a significantly lower survival rate than ground squirrels $(P=0.015)$, with only two of ten rats maintaining spontaneous ventilation for $60 \mathrm{~min}$ of hypoxia. When surviving rats and ground squirrels were sacrificed during normoxia (control) and at 5, 10, and $60 \mathrm{~min}$ of hypoxic exposure, $\beta$-hydroxybutyrate (BHB) levels were significantly higher in ground squirrels than in rats (Fig. 2). BHB levels in both rats and ground squirrels increased during

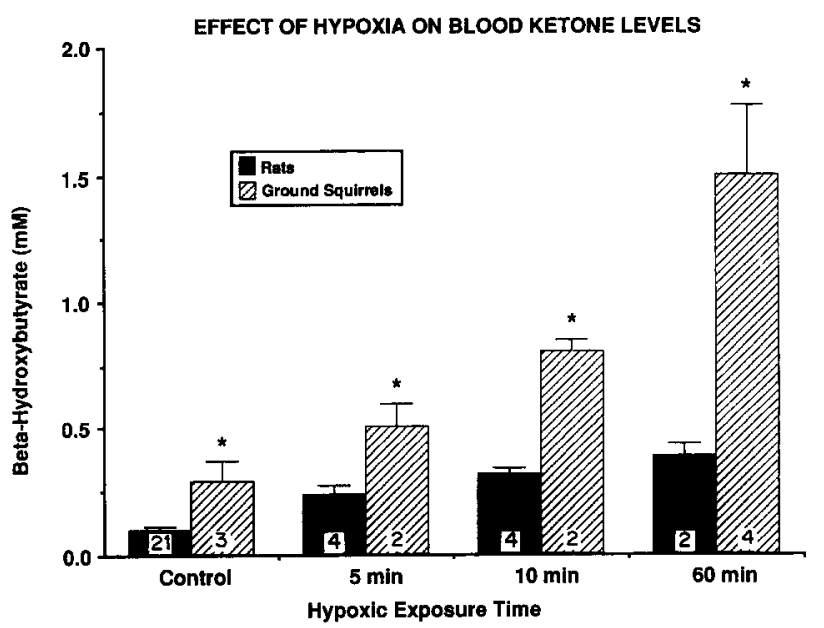

Fig. 2. Whole blood $\beta$-hydroxybutyrate (BHB) concentration in non-hibernating ground squirrels and rats before (control) and during hypoxia $\left(4.5 \% \mathrm{O}_{2}\right)$. The four groups consist of separate animals that were sacrificed at the specified times. Ground squirrels had significantly higher BHB levels in each of the four comparisons $\left({ }^{*} P \leq 0.05\right.$, Student's $t$-test $)$. Values are given $\pm S E M$ with sample sizes indicated inside each bar. 
BLOOD BETA-HYDROXYBUTYRATE LEVELS VS SURVIVAL IN RATS

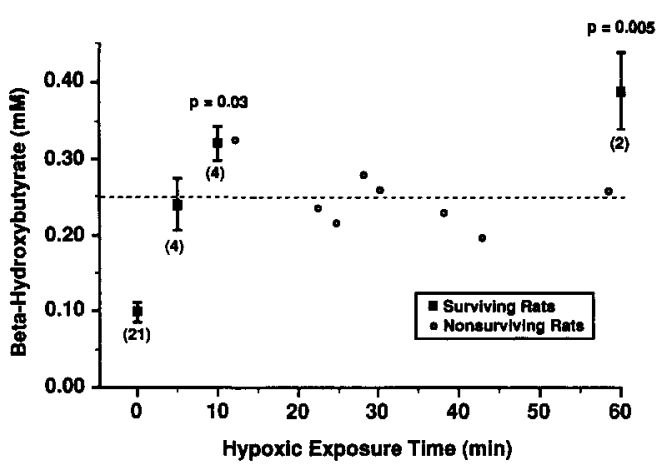

Fig. 3. Whole blood $\beta$-hydroxybutyrate (BHB) concentration in control rats and rats exposed to hypoxia $\left(4.5 \% \mathrm{O}_{2}\right)$. Squares symbolize rats killed, and circles represent rats which died during hypoxia at the indicated time. Sample sizes are given below each group. Compared to the mean BHB level of non-surviving rats (horizontal broken line), surviving rats at the 10 and $60 \mathrm{~min}$ times had significantly higher BHB levels (Student's $t$-test).

exposure to hypoxia but the percentage and magnitude of the increase was much greater in the ground squirrels. Over the $60 \mathrm{~min}$ hypoxic period, blood BHB increased $517 \%$ in ground squirrels compared to $378 \%$ in rats. Figure 3 indicates that for the four rats decapitated after surviving $10 \mathrm{~min}$ of hypoxia and for the two rats decapitated after surviving at $60 \mathrm{~min}$, blood BHB levels were significantly elevated above the mean BHB level of the eight non-surviving rats.

The second set of experiments involved comparison between hibernating and non-hibernating ground squirrels. BHB levels were significantly increased in hibernating ground squirrels (Table 1). In contrast, plasma glucose concentrations were significantly decreased in hibernating ground squirrels and plasma concentrations of glucagon and insulin tended to be lower.
To test the hypothesis that cold exposure itself would elevate blood BHB levels, rats and ground squirrels were exposed to $10^{\circ} \mathrm{C}$ for $48 \mathrm{hr}$. There was a tendency for BHB blood levels to increase, but when compared to values in animals housed at $22^{\circ} \mathrm{C}$, the difference was not significant (Table 2).

When isolated mitochondria oxidative phosphorylation was tested at $13^{\circ} \mathrm{C}$ (the average body temperature of hibernating ground squirrels) with BHB as substrate there was no difference in mitochondrial function between hibernating and non-hibernating ground squirrels (data not shown). Figure 4 shows that when mitochondria were tested at $37^{\circ} \mathrm{C}$ with either BHB as the sole substrate (top panel), or with a combination of glutamate and malate as substrate (bottom panel), the mitochondria from hibernating ground squirrels had reduced oxidative phosphorylation rates and respiratory control ratios, respectively, compared to non-hibernating ground squirrels.

\section{DISCUSSION}

Experimental fasting, alloxan diabetes (Kirsch and D'Alecy, 1979) and 1,3-butanediol treatment (Kirsch et al., 1980), all of which induce ketosis, were associated with a dramatically increased hypoxic tolerance in the mouse. In the Levine rat preparation, 1,3-butanediol treatment elevated blood BHB and correlated with a significant increase in hypoxic survival time (Lundy et al., 1984). Although there is a strong correlation between experimental ketosis and increased hypoxic survival time, the mechanism for this protection is unknown. In an attempt to establish a cause and effect relationship, studies have directly elevated blood ketones by injection of $\beta$-hydroxybutyrate (BHB). Eiger et al. (1980) found that BHB plus glucagon treatment, but not BHB alone, was associated with increased hypoxic tolerance, and hypothesized that glucagon may have a permissive role for BHB action. Lundy et al. (1987) found no correlation between treatment with BHB and glucagon in the

Table 1. Comparisons between non-hibernating and hibernating ground squirrels

\begin{tabular}{lccc}
\hline & Non-hibernating & Hibernating & Student's- $t$-test \\
\hline Weight $(\mathrm{g})$ & $162 \pm 10(n=18)$ & $111 \pm 11(n=17)$ & $P=0.0016$ \\
Body temperature $\left({ }^{\circ} \mathrm{C}\right)$ & $37.3 \pm 0.3(n=17)$ & $13 \pm 4(n=17)$ & $P<0.0001$ \\
HCT $(\%)$ & $51.2 \pm 1.3(n=11)$ & $43.6 \pm 3.8(n=10)$ & $P=0.067$ \\
BHB $(\mathrm{mM})$ & $0.46 \pm 0.1(n=18)$ & $1.6 \pm 0.3(n=17)$ & $P=0.0005$ \\
Glucose $(\mathrm{mg} / \mathrm{dl})$ & $153 \pm 7(n=18)$ & $75 \pm 7(n=15)$ & $P<0.0001$ \\
Glucagon $(\mathrm{pg} / \mathrm{ml})$ & $445 \pm 11(n=7)$ & $175 \pm 37(n=6)$ & $P=0.056$ \\
Insulin $(\mathrm{uu} / \mathrm{ml})$ & $16.5 \pm 6.5(n=7)$ & $10.4 \pm 2.5(n=6)$ & $P=0.43$ \\
\hline
\end{tabular}

Non-hibernating ground squirrels were housed at $10^{\circ} \mathrm{C}$ for $48 \mathrm{hr}$ to control for temperature effect of housing hibernating ground squirrels at $10^{\circ} \mathrm{C}$. BHB, glucose, glucagon and insulin are from whole blood samples.

Values are given \pm SEM.

Table 2. Ketone levels in rats and non-hibernating ground squirrels

\begin{tabular}{llll}
\hline & \multicolumn{1}{c}{$22^{\circ} \mathrm{C}$} & \multicolumn{1}{c}{$48 \mathrm{hr}$ at $10^{\circ} \mathrm{C}$} & Student's $t$-test \\
\hline Rat blood BHB (mM) & $0.10 \pm 0.01(n=21)$ & $0.15 \pm 0.03(n=16)$ & $P=0.12$ \\
Ground squirrel blood BHB (mM) & $0.29 \pm 0.08(n=3)$ & $0.46 \pm 0.1(n=18)$ & $P=0.51$ \\
\hline
\end{tabular}

Whole blood $\beta$-hydroxybutryate (BHB) concentration in rats and ground squirrels exposed to room temperature or cold stress $\left(10^{\circ} \mathrm{C}\right.$ for $\left.48 \mathrm{hr}\right)$.

Values are given \pm SEM. 
MITOCHONDRIAL FUNCTION
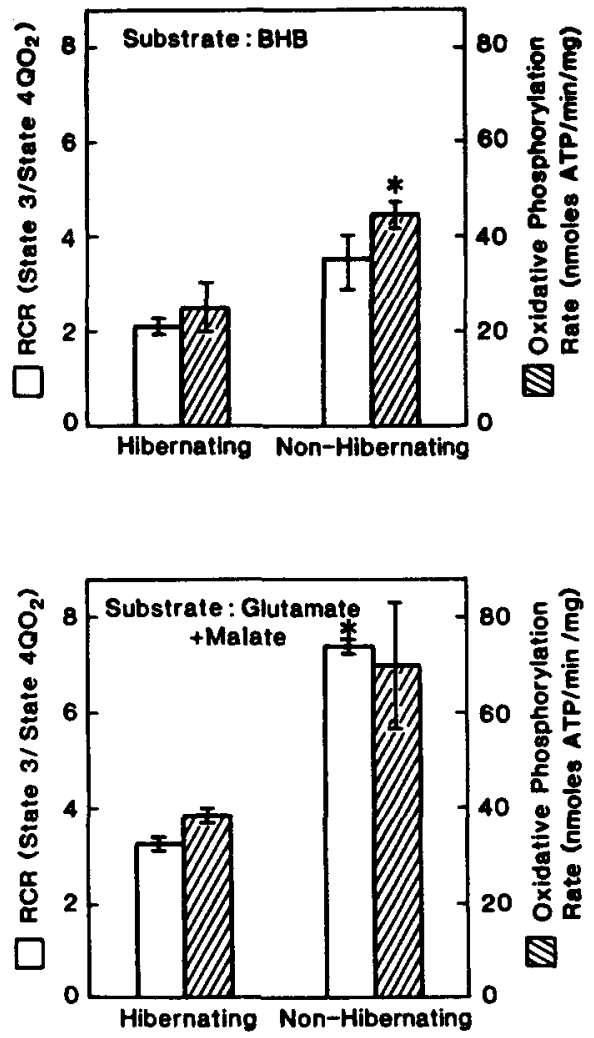

Fig. 4. Respiratory control ratios (RCR) and oxidative phosphorylation rates (OPR) in hibernating and non-hibernating ground squirrel heart mitochondria at $37^{\circ} \mathrm{C}$. With either BHB as the sole substrate (top panel), or with a combination of glutamate and malate as substrate (bottom panel), the mitochondria from hibernating ground squirrels had reduced oxidative phosphorylation rates and respiratory control ratios, respectively. $\left({ }^{*} P \leq 0.05\right.$, Student's $t$-test). Values are given at $\pm \mathrm{SEM}$.

Levine rat but suggested that an induction period for brain BHB metabolism may be required.

Even though a causal role for ketones and hypoxic survival time has not been demonstrated, the strong association merits further investigation. As an alternative approach to examining the role of ketones in hypoxia under artificial experimental conditions, we chose an animal model that naturally encounters hypoxic conditions. A strong association between $\mathrm{BHB}$ and increased hypoxic tolerance under natural conditions would not establish a causal role for BHB but would lend additional support for an important basic biological role for BHB during hypoxia. It would not only be an additional animal model demonstrating this association but also a naturally occurring biological change with potential adaptive significance. Hibernators such as the ground squirrel are exposed to moderate hypoxia when metabolism increases rapidly and tissue oxygen tension falls during arousal from the hibernating state. Because hibernating animals arouse and return to hibernation frequently during the total hibernation period they are often exposed to hypoxic conditions (Baumber et al., 1971). We hypothesized that because exogenously induced systemic ketosis was protective in laboratory rodents when exposed to hypoxia, hibernating rodents may benefit from a natural endogenous induction of ketosis.

The hypoxic survival rate of ground squirrels was significantly higher than rats. Blood BHB concentration in ground squirrels underwent a larger percentage increase and reached significantly higher absolute levels than in rats. Although rats had a significant increase in blood BHB, the absolute levels to which they rose may not have been as protective. In addition, rats that survived the longest had the highest BHB levels, indicating that elevated BHB may have been beneficial.

During hibernation, metabolic rates in ground squirrels are $2 \%$ of normothermic rates (Deavers and Musacchia, 1980). Table 1 compares the variables measured in non-hibernators and hibernators. As expected, weight and body temperature are significantly lower in hibernators. Consistent with the picture of starvation seen in man, the blood glucose level is significantly lower in hibernators, and the BHB levels are significantly higher. As with human starvation, hibernators shift from glucose to ketone bodies as a primary fuel source. Studies have shown that ketones lead to glucose sparing in peripheral tissues (Robinson and Williamson, 1980; Krilowicz 1985). This might suggest that the spared glucose can then be utilized preferentially by the central nervous system, but other studies (Kirsch and D'Alecy, 1984) have shown that the brain will preferentially use BHB over glucose. Therefore a potentially protective mechanism of BHB may be as an energy source which cannot be metabolized into lactate during hypoxic conditions.

Mitochondrial function was examined in nonhibernating and hibernating ground squirrels at $37^{\circ} \mathrm{C}$ in an attempt to determine if hibernating ground squirrels, which are exposed to natural hypoxic conditions with resultant ketosis, have preferential BHB utilization. This was done by determining the respiratory control ratio and the oxidative phosphorylation rate for isolated heart mitochondria. When tested at the body temperature of hibernating ground squirrels $\left(13^{\circ} \mathrm{C}\right)$, no difference in mitochondrial function was observed. When isolated mitochondria were tested at $37^{\circ} \mathrm{C}$ with $\mathrm{BHB}$ as the sole substrate, mitochondria from hibernating ground squirrels had reduced oxidative phosphorylation rates (Fig. 4). With a combination of glutamate and malate as substrate, the mitochondria from hibernating ground squirrels had reduced respiratory control ratios compared to nonhibernating ground squirrels. These data indicate no preferential BHB utilization by mitochondria isolated from hibernating ground squirrel hearts and so offer no direct support for a causal role for BHB increasing tolerance to hypoxia. Indeed, there appears to be a depression of mitochondrial function with hibernation that is not immediately reversed by temperature. These results might suggest that if BHB plays a role in increasing tolerance to hypoxia it may be via extramitochondrial factors, and that hibernation produces mitochondrial changes not readily reversed by increasing temperature. In addition, the mitochondrial function of the heart does not necessarily reflect that within the brainstem which is the most likely site 
of hypoxic intolerance as measured in this study (ventilatory arrest).

Since exposure of rats to $10^{\circ} \mathrm{C}$ did not result in a significant increase in blood BHB concentration, it is not likely that reduced ambient temperature itself caused the higher blood BHB concentrations in ground squirrels. Thus hypoxia is probably the more likely trigger of $\mathrm{BHB}$ mobilization. This finding is consistent with recent experiments which have shown that decreased body temperature could only partially account for BHB mobilization in hypoxic mice and that hypoxia is the major stimulus (Rising and D'Alecy, 1989).

In summary, non-hibernating ground squirrels exposed to hypoxia show a marked increase in blood BHB and survive at least $1 \mathrm{hr}$. This level of hypoxia was shown in previous studies to kill mice in $2 \mathrm{~min}$ and in the current study to kill $80 \%$ of rats in an hour. Only those rats that maintain an increase in blood BHB survived hypoxia for up to $1 \mathrm{hr}$. Therefore, endogenous as well as exogenous elevation of blood BHB levels appears to be associated with increased hypoxic tolerance. In hibernating ground squirrels, blood BHB is elevated, further suggesting that ketosis may have evolved as an adaptation to life threatening hypoxia.

\section{REFERENCES}

Baumber J., South F. E., Ferren L. and Zatzman M. L. (1971) A possible basis for periodic arousals during hibernation: accumulation of ketone bodies. Life Sci. 10, 463-467.

Bullard R. W., David G. and Nichols C. T. (1960) The mechanism of hypoxic tolerance in hibernating and nonhibernating mammals. Bull. Mus. comp. Zool. 124, 321-336.

Chance B. and Williams G. R. (1956) The respiratory chain and oxidative phosphorylation. Adv. Enzymol. 17, 65-134.

D'Alecy L. G., Myers C. L., Brewer M., Rising C. L. and Shlafer M. (1986) Substrate-specific stimulation by glucagon of isolated murine brain mitochondrial oxidative phosphorylation. Stroke 17, 305-312.

Deavers D. R. and Musacchia X. J. (1980) Water metabolism and renal function during hibernation and hypothermia. Fed. Proc. 39, 2969-2973.
Eiger S. M., Kirsch J. R. and D'Alecy L. G. (1980) Hypoxic tolerance enhanced by beta-hydroxybutyrate-glucagon in the mouse. Stroke 11, 513-517.

Gottstein U., Held K., Muller W. and Berghoff W. (1972) Utilization of ketone bodies by the human brain. In Research on Cerebral Circulation (Edited by Meyer J. S., Reivich M., Lechner H. and Eichhorn O.) pp. 137-145. Fifth International Salzburg Conference, Springfield, IL. Hiestand W. A., Rockhold W. T. Stemler R. W., Stullken D. E. and Wiebers J. E. (1950) The comparative hypoxic resistance of hibernators and non-hibernators. Physiol. Zool. 23, 264-268.

Himwich H. E. Bernstein A. O., Herrlich H., Chesler A. and Fazekas J. F. (1942) Mechanisms for the maintenance of life in the newborn during anoxia Am. J. Physiol. 135, 387-391.

Kirsch J. R. and D'Alecy L. G. (1979) Effect of altered availability of energy-yielding substrates upon survival from hypoxia in mice. Stroke 10, 288-291.

Kirsch J. R. and D'Alecy L. G. and Mongroo P. B. (1980) Butanediol induced ketosis increases tolerance to hypoxia in the mouse. Stroke 11, 506-512.

Kirsch J. R., D'Alecy L. G. (1984) Hypoxia induced preferential ketone utilization by rat brain slices. Stroke 15, 319-323.

Krilowicz B. L. (1985) Ketone body metabolism in a ground squirrel during hibernation and fasting. Am. J. Physiol. 249R, 462-470.

Lundy E. F., Luyckx B. A., Zelenock G. B. and D'Alecy L. G. (1984) Butanediol induced cerebral protection in the instrumented Levine rat. Stroke 15, 547-552.

Lundy E. F., Klima L. D., Huber T. S., Zelenock G. B. and D'Alecy L. G. (1987) Elevated blood ketone and glucagon levels cannot account for 1,3-butanediol induced cerebral protection in the Levine rat. Stroke 18, 217-222.

Lyman C. P., Willis J. S., Malan A. and Wang L. D. H. (1982) Hibernation and Torpor in Mammals and Birds, pp. 159, 271-272. Academic Press, New York.

Owen O. E. Morgan A. P., Kemp H. G., Sullivan J. M., Herrara M. G. and Cahill G. F. (1987) Brain metabolism during fasting. J. clin. Invest. 46, 1589-1595.

Rising C. L. and D'Alecy L. G. (1989) Acute adaption to hypoxia augmented by beta-hydroxybutryate in the mouse. Stroke 20, 1219-1225.

Robinson A. M. and Williamson D. H. (1980) Physiological roles of ketone bodies as substrates and signals in mammalian tissues. Physiol. Rev. 60, 143-87.

US Department of Health and Human Services (1985) Guide for the Care and Use of Experimental Animals, Publication No. $86-23$. 